\title{
Calcium efflux from the endoplasmic reticulum regulates cisplatin-induced apoptosis in human cervical cancer HeLa cells
}

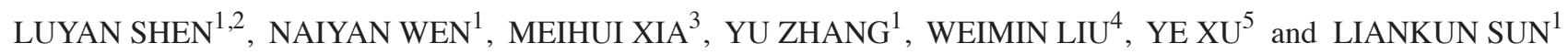 \\ ${ }^{1}$ Department of Pathophysiology, Basic College of Medicine; ${ }^{2}$ Department of Obstetrics and Gynecology, Second Hospital; \\ ${ }^{3}$ Department of Obstetrics and Gynecology, First Hospital, Jilin University, Changchun, Jilin 130021; \\ ${ }^{4}$ Department of Interventional Radiology, Renmin Hospital; ${ }^{5}$ Medical Research Laboratory, \\ Jilin Medical College, Jilin, Jilin 132013, P.R. China
}

Received December 31, 2014; Accepted February 4, 2016

DOI: $10.3892 / 01.2016 .4278$

\begin{abstract}
The function of calcium efflux from the endoplasmic reticulum (ER) in cisplatin-induced apoptosis is not fully understood in cancer cells. The present study used western blot analysis, flow cytometry, immunofluorescence and 3-[4,5-dimethylthiazol-2-yl]-2,5 diphenyl tetrazolium bromide assay to investigate calcium signaling in human cervical cancer cells exposed to cisplatin. In the present study, treatment with cisplatin increased free $\mathrm{Ca}^{2+}$ levels in the cytoplasm and mitochondria of human cervical cancer HeLa cells, which further triggers the mitochondria-mediated and ER stress-associated apoptosis pathways. Notably, blocking calcium signaling using the calcium chelating agent bis-(o-aminophenoxy)ethane-N,N,N',N'-tetra-acetic acid acetoxymethyl ester inhibited cisplatin-induced apoptosis via downregulation of the calcium-dependent proteases, the calpains, and innate apoptosis proteins, such as caspsae-3, caspase-4 and C/EBP homologous protein (CHOP). In addition, use of the inositol triphosphate receptor inhibitor, 2-aminoethyl diphenylborinate, to inhibit calcium efflux from the ER resulted in similar effects. This data indicated that calcium efflux from the ER plays a significant role in cisplatin-induced apoptosis in human cervical cancer HeLa cells, which provides further mechanistic insights into the tumor cell-killing effect of cisplatin and potential therapeutic strategies to improve cisplatin chemotherapy.
\end{abstract}

Correspondence to: Dr Ye Xu, Medical Research Laboratory, Jilin Medical College, 5 Jilin Street, Jilin, Jilin 132013, P.R. China

E-mail: xuye_9707@163.com

Dr Liankun Sun, Department of Pathophysiology, Basic College of Medicine, Jilin University, 126 Xinmin Street, Changchun, Jilin 130021, P.R. China

E-mail: sunlk@jlu.edu.cn

Key words: calcium, cisplatin, apoptosis, endoplasmic reticulum stress, cervical cancer

\section{Introduction}

Cisplatin (cis-diamminedichloroplatinum II) is one of the clinical chemotherapeutic agents used against a broad spectrum of human malignancies, such as ovarian, cervical, prostate and lung cancer (1). However, relapse following cisplatin therapy is inevitable. Thus, it is particularly important to elucidate the cell-killing mechanism of cisplatin (2-4). Cisplatin is generally considered to kill cancer cells by damaging DNA and inhibiting DNA synthesis, which induces mitochondria-mediated apoptosis and consequently, cell death (5-8). Recent findings have revealed that cisplatin triggers apoptotic events via endoplasmic reticulum (ER) stress $(9,10)$.

The ER is a crucial organelle in eukaryotic cells that is essential for the biological processes required for cell survival and normal cell function, such as protein folding and secretion, lipid biosynthesis and calcium homeostasis. Multiple stimuli can cause the accumulation of unfolded and incompletely folded proteins in the ER, which initiates the unfolded protein response (UPR) and ER stress $(11,12)$. In the resting state, the $\mathrm{ER}$, an intracellular store of calcium, regulates basic calcium oscillations via calcium ion channels on the ER membrane in order to transmit intracellular biological information. Once balance is disrupted, a regulatory network of downstream signaling pathways is activated (13-15). Previous studies have suggested that chemotherapeutic agents target the metabolism of reactive oxygen species and ATP production in the mitochondria, activate mitochondria-mediated cell apoptosis, and inhibit cell growth and proliferation via an increase in cellular calcium mobilization (16-18). However, the function of calcium signaling in ER-derived apoptosis induced by chemotherapy drugs is not clear.

Thus, we presume that calcium signaling plays an important role in the mitochondria-mediated and ER-mediated apoptosis pathways following cisplatin exposure in human cervical cancer HeLa cells. In the present study, it was found that treatment with cisplatin significantly increased cellular calcium concentrations and triggered the mitochondria-dependent and ER-dependent apoptosis pathways in HeLa cells, which could be inhibited by blocking calcium signaling. These results demonstrated that calcium efflux from the ER plays a significant role in the regulation of apoptosis triggered by cisplatin in HeLa cells. 


\section{Materials and methods}

Reagents and antibodies. In the present study, the cisplatin, bis-(o-aminophenoxy)ethane-N,N,N',N'-tetra-acetic acid acetoxymethyl ester (BAPTA/AM), inositol triphosphate receptor (IP3R) inhibitor 2-aminoethyl diphenylborinate (2-APB) and 3-(4,5-dimetrylthiazol-2-yl)-2,5-diphenyltetrazolium bromide (MTT) were purchased from Sigma-Aldrich (St. Louis, MO, USA). Fetal bovine serum (FBS) and Iscove's modified Dulbecco's medium (IMDM) were purchased from Life Technologies (Thermo Fisher Scientific Inc., Waltham, MA, USA). Fluo-4/AM, Alexa Fluor 546 donkey polyclonal anti-rabbit immunoglobulin (Ig)G (catalog no., A10040), Alexa Fluor 546 donkey polyclonal anti-mouse IgG (catalog no., A10036) and Alexa Fluor 488 donkey polyclonal anti-rabbit $\operatorname{IgG}$ (catalog no., A-21206) were purchased from Invitrogen (Thermo Fisher Scientific Inc.). Rhod-2/AM was purchased from AAT Bioquest Inc. (Sunnyvale, CA, USA). Enhanced chemiluminescence (ECL) reagents were obtained from Thermo Fisher Scientific Inc. Mouse monoclonal anti-C/EBP homologous protein (CHOP; catalog no., ab11419), rabbit polyclonal anti-caspase-3 (catalog no., ab13847) and rabbit polyclonal anti-activated-caspase-3 (catalog no., ab2302) antibodies were purchased from Abcam (Cambridge, MA, USA), while rabbit polyclonal anti-caspase-4 (catalog no., 24287) and rabbit polyclonal anti-calpain-1 catalytic subunit (CAPN1; catalog no., 32201) antibodies were purchased from Signalway Antibody Co. (College Park, MD, USA), and mouse monoclonal anti-glucose-regulated protein (GRP78; $78 \mathrm{kDa}$; catalog no., sc-376768) antibody was purchased from Santa Cruz Biotechnology Inc. (Dallas, TX, USA). Rabbit polyclonal anti- $\beta$-actin (catalog no., 20536-1-AP) and peroxidase-conjugated Affinipure goat anti-mouse- and anti-rabbit Ig were purchased from Proteintech Group Inc. (Chicago, IL, USA; catalog nos., SA00001-1 and SA00001-2). All other reagents and antibodies were purchased from Changchun Baoxin Biotechnology Co. (Changchun, China).

Cell culture. Human cervical cancer HeLa cells were obtained from China Academy of Chinese Medical Sciences (Beijing, China), and were cultured at $37^{\circ} \mathrm{C}$ in a $5 \%(v / v) \mathrm{CO}_{2}$ and 95\% (v/v) air atmosphere, using IMDM containing $10 \%(\mathrm{v} / \mathrm{v})$ FBS, and $100 \mathrm{U} / \mathrm{ml}$ penicillin and $100 \mu \mathrm{g} / \mathrm{ml}$ streptomycin. The cells were divided into six groups: Non-treated cells, cells treated with $5 \mu \mathrm{g} / \mathrm{ml}$ cisplatin, cells treated with $2.5 \mu \mathrm{M}$ BAPTA/AM, cells treated with $100 \mu \mathrm{M} 2-\mathrm{APB}$, cells treated with $5 \mu \mathrm{g} / \mathrm{ml}$ cisplatin combined with $2.5 \mu \mathrm{M}$ BAPTA/AM, and cells treated with $5 \mu \mathrm{g} / \mathrm{ml}$ cisplatin combined with $100 \mu \mathrm{M} 2$-APB.

Determination of intracellular and mitochondrial free $\mathrm{Ca}^{2+}$ levels. The fluorescent calcium indicator Fluo-4/AM $(5 \mu \mathrm{M})$ and Rhod-2 $(5 \mu \mathrm{M})$ were used to measure intracellular and mitochondrial free $\mathrm{Ca}^{2+}$ levels, as previously described (19). The cells were monitored using an Olympus FV1000 confocal laser microscope (Olympus Corporation, Tokyo, Japan). Fluorescence images labeled with Fluo-4/Rhod-2 were collected using an excitation wavelength of $488 / 546 \mathrm{~nm}$. The same parameters of illumination and detection were maintained digitally for consistency throughout the experiments.
MTT assay. Cell viability was determined using the MTT assay. In the experiments, exponentially growing HeLa cells were seeded in 96 -well culture plates at a density of $1 \times 10^{4}$ cells/well. After $24 \mathrm{~h}$ of incubation, cisplatin with or without BAPTA/AM and 2-APB was added for $24 \mathrm{~h}$ in four parallel wells. MTT solution $(5 \mathrm{mg} / \mathrm{ml})$ was added for $4 \mathrm{~h}$, followed by the addition of $150 \mu \mathrm{l}$ dimethyl sulfoxide. After $10 \mathrm{~min}$ of shaking, the absorbance was measured at $570 \mathrm{~nm}$ using a microplate reader (iMark; Bio-Rad Laboratories, Inc., Hercules, CA, USA). The survival rate was calculated as follows: Survival $(\%)=$ absorbance of experimental group / absorbance of control group x 100. In each experiment, the mean value of four wells per treatment group was calculated.

Flow cytometry analysis. The MUSE ${ }^{\mathrm{TM}}$ Annexin-V Dead Cell kit (EMD Millipore, Billerica, MA, USA) was used to monitor cell death. In the experiments, exponentially growing HeLa cells were seeded in 6-well culture plates at a density of $2 \times 10^{5}$ cells/well. Following exposure to the different experimental conditions, the cells were trypsinized and resuspended in IMDM medium with $10 \%$ FBS at a concentration of $1 \times 10^{6}$ cells $/ \mathrm{ml}$. The cells were incubated with Annexin-V in the dark at room temperature for $20 \mathrm{~min}$. Finally, samples were detected using the MUSE Cell Analyzer (EMD Millipore). All experiments were performed in triplicate.

Immunofluorescence staining and confocal laser microscopy. The cells were cultured on coverslips overnight, and after treatment with the indicated dose of cisplatin with or without BAPTA/AM and 2-APB for $24 \mathrm{~h}$, were fixed with $4 \%(\mathrm{w} / \mathrm{v})$ paraformaldehyde, stained with nuclear Hoechst $33342(1 \mu \mathrm{g} / \mathrm{ml}$; Sigma-Aldrich) for $5 \mathrm{~min}$, washed with phosphate-buffered solution (PBS), and examined using an Olympus FV1000 confocal laser microscope to reveal cell chromatin condensation. The expression of GRP78, active caspase- 3 and CHOP was examined by indirect immunofluorescence. The cells were cultured on coverslips overnight, then treated with the indicated dose of cisplatin with or without BAPTA/AM and 2APB for $24 \mathrm{~h}$, and rinsed with PBS three times. After incubation, the cells were fixed with $4 \%(\mathrm{w} / \mathrm{v})$ paraformaldehyde for $20 \mathrm{~min}$, permeabilized with $0.1 \%$ (v/v) Triton X-100 for $5 \mathrm{~min}$, blocked with bovine serum albumin, and incubated with the primary antibodies for GRP78 (1:50 dilution), active caspase-3 (1:250 dilution), and CHOP (1:100 dilution) overnight at $4^{\circ} \mathrm{C}$. The cells were then incubated in Alexa Fluor 488 Donkey Anti-Rabbit IgG, Alexa Fluor 546 Donkey Anti-Rabbit IgG and Alexa Fluor 546 Donkey Anti-Mouse IgG secondary antibodies (1:400 dilution) for $30 \mathrm{~min}$, stained with Hoechst 33342 $(1 \mu \mathrm{g} / \mathrm{ml})$ for $5 \mathrm{~min}$ and washed with PBS three times. After mounting, the cells were examined under an Olympus FV1000 confocal laser microscope. The same parameters of illumination and detection were maintained digitally throughout the experiments.

Western blotting. Whole-cell protein extracts from the HeLa cells were prepared with cell lysis buffer [50 mM Tris-HCl (pH 7.5), $150 \mathrm{mM} \mathrm{NaCl}, 1 \mathrm{mM}$ sodium-EDTA, $1 \mathrm{mM}$ EDTA, $1 \%$ (v/v) Triton X-100, $2.5 \mathrm{mM}$ sodium pyrophosphate, $1 \mathrm{mM}$ $\beta$-glycerophosphate, $1 \mathrm{mM} \mathrm{Na}_{3} \mathrm{VO}_{4}, 1 \mathrm{mM} \mathrm{NaF}, 1 \mu \mathrm{g} / \mathrm{ml}$ leupeptin and $1 \mathrm{mM}$ PMSF] for western blotting. Protein 


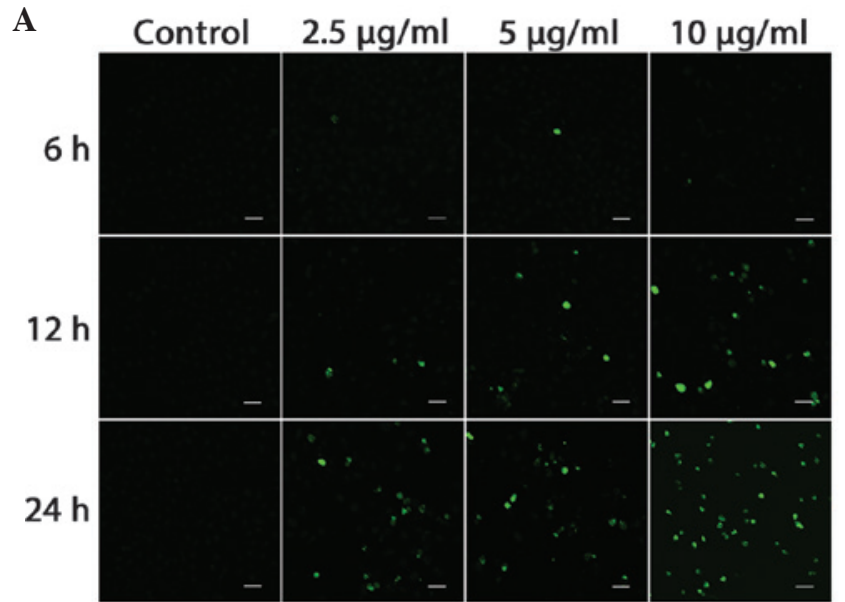

B
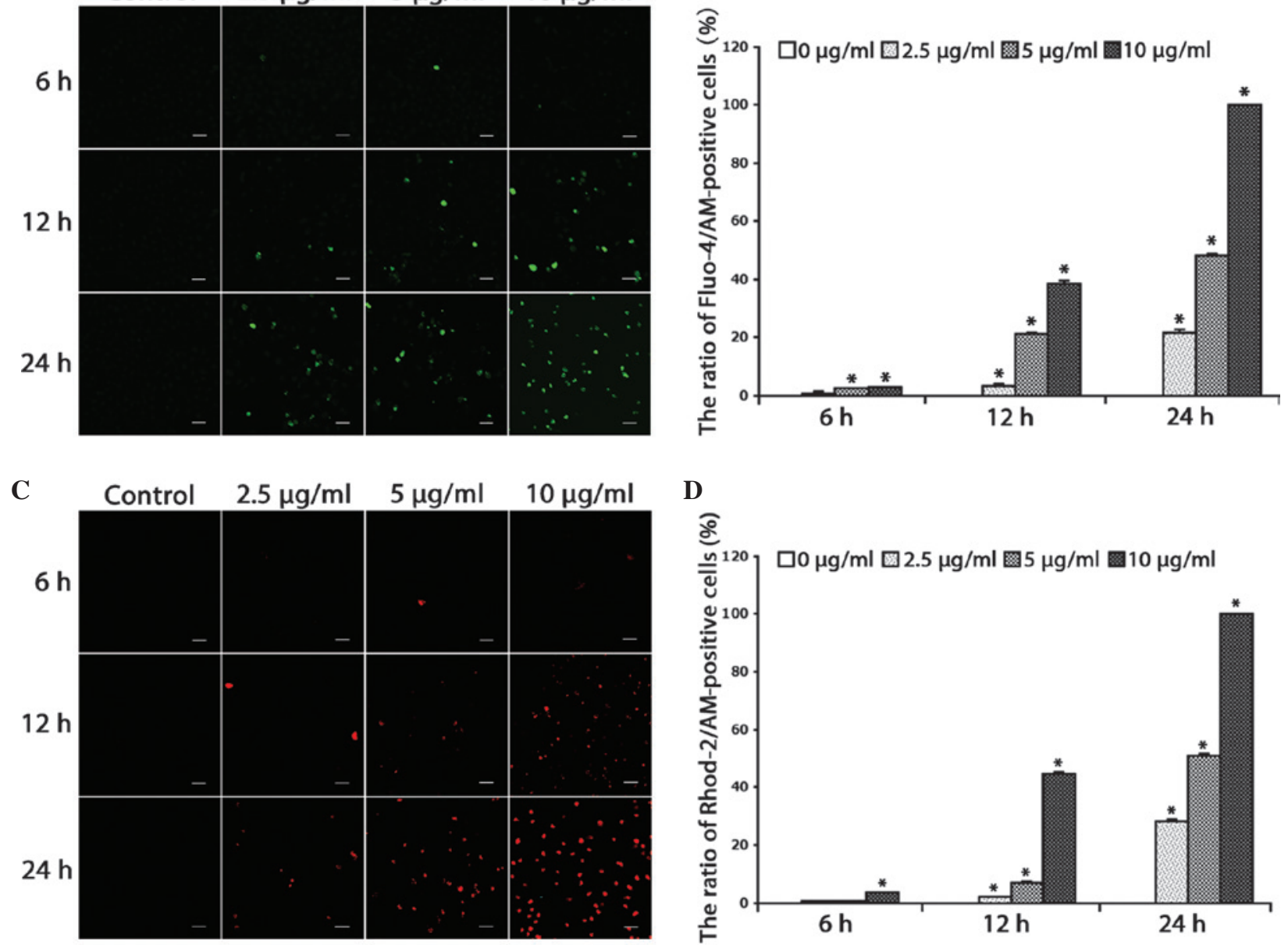

Figure 1. Cisplatin increases free $\mathrm{Ca}^{2+}$ levels in the cytosol and mitochondria. (A) The cells were treated with cisplatin $(2.5,5$ or $10 \mu \mathrm{g} / \mathrm{ml})$ for $0,6,12$ and $24 \mathrm{~h}$, and incubated with the fluorescent calcium indicator, Fluo-4/AM. Calcium concentrations in the cytosol were observed by confocal microscopy (scale bar, $40 \mu \mathrm{m})$. (B) Quantitation of free $\mathrm{Ca}^{2+}$ levels in the cytosol. Data are presented as the mean $\pm \mathrm{SD}(\mathrm{n}=3)$. ${ }^{*} \mathrm{P}<0.05$ vs. control. (C) Cells were treated with cisplatin $(2.5,5$ or $10 \mu \mathrm{g} / \mathrm{ml})$ for $0,6,12$ and $24 \mathrm{~h}$, and incubated with the fluorescent calcium indicator, Rhod-2. Calcium concentrations in the mitochondria were observed by confocal microscopy (scale bar, $40 \mu \mathrm{m}$ ). (D) Quantitation of free $\mathrm{Ca}^{2+}$ levels in the mitochondria. Data are presented as the mean $\pm \mathrm{SD}(\mathrm{n}=3)$. ${ }^{*} \mathrm{P}<0.05$ vs. control. SD, standard deviation.

concentration was quantified using the BCA Protein Assay kit (Pierce ${ }^{\mathrm{TM}}$; Thermo Fisher Scientific Inc.). For western blot analysis, lysates $(30 \mu \mathrm{g})$ were resolved on $10 \%(\mathrm{w} / \mathrm{v})$ sodium dodecyl sulfate-polyacrylamide gels and transferred onto immobilon-P transfer membranes (EMD Millipore). The membranes were blocked with $5 \%(\mathrm{w} / \mathrm{v})$ skimmed dry milk in buffer [10 mM Tris- $\mathrm{HCl}(\mathrm{pH} 7.6), 100 \mathrm{mM} \mathrm{NaCl}$ and $0.1 \%$ Tween 20] for $1 \mathrm{~h}$ at room temperature and then incubated with the desired primary antibody overnight at $4^{\circ} \mathrm{C}$, followed by incubation with horseradish peroxidase-conjugated secondary antibody (1:2,000; Proteintech Group Inc.) for $1.5 \mathrm{~h}$ at room temperature. Immunodetection was performed using the ECL reagents and images were captured using Syngene Bio Imaging (Synoptics, Cambridge, UK). The level of protein was normalized to that of actin and the ratios of normalized protein to actin are presented as the mean \pm standard deviation from three independent experiments. Protein levels were quantified by densitometry using Quantity One version 4.6.2 software (Bio-Rad Laboratories Inc.).

Statistical analysis. Data are representative of three independent experiments each performed in triplicate. Statistical analysis of the data was performed using a one-way analysis of variance on IBM SPSS version 22.0 (IBM SPSS, Armonk, NY, USA). Tukey's post-hoc test was used to determine the significance for all pairwise comparisons of interest. $\mathrm{P}<0.05$ was considered to indicate a statistically significant difference.

\section{Results}

Cisplatin increases free $\mathrm{Ca}^{2+}$ levels in the cytosol and mitochondria. For the determination of cytosolic and mitochondrial free $\mathrm{Ca}^{2+}$ levels, the $\mathrm{Ca}^{2+}$-sensitive fluorescent dyes, Fluo-4/AM and Rhod-2/AM, were used. Based on previous studies (10), the HeLa cells were treated with increasing doses of cisplatin (2.5, 5 and $10 \mu \mathrm{g} / \mathrm{ml}$ ) for $0,6,12$ and $24 \mathrm{~h}$, and further incubated with the calcium indicators Fluo-4/AM and Rhod-2/AM. Confocal microscopy was used to observe alterations in free $\mathrm{Ca}^{2+}$ levels. It was found that the free $\mathrm{Ca}^{2+}$ levels in the cytosol (Fig. 1A and B) and mitochondria (Fig. 1C and D) increased in a dose- and time-dependent manner in the HeLa cells $(\mathrm{P}<0.001)$.

These results suggested that cisplatin increases free $\mathrm{Ca}^{2+}$ levels in the cytosol and mitochondria of HeLa cells, indicating the relevance of calcium in the cell death induced by cisplatin. 
A

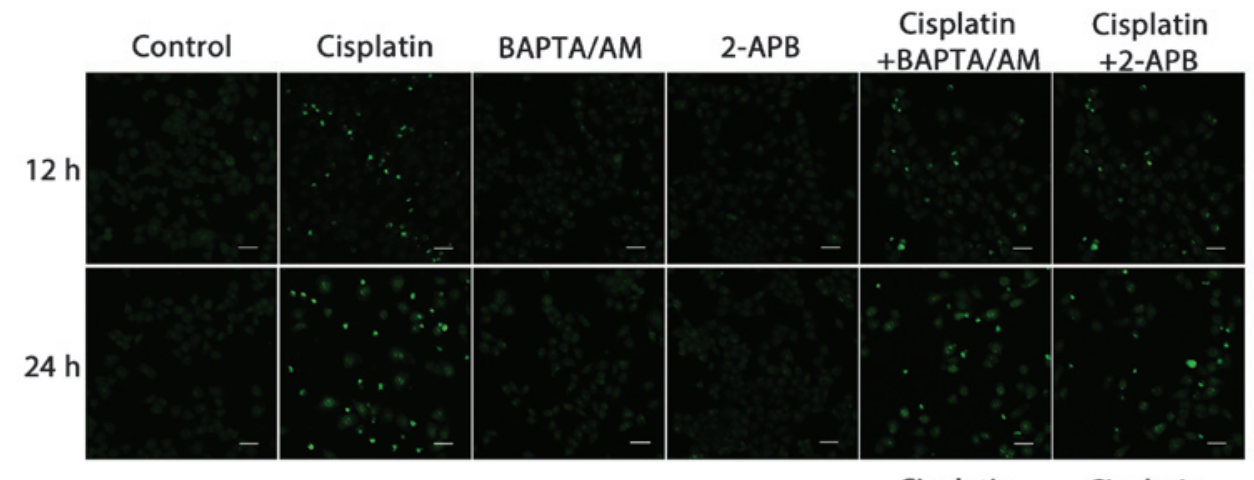

B

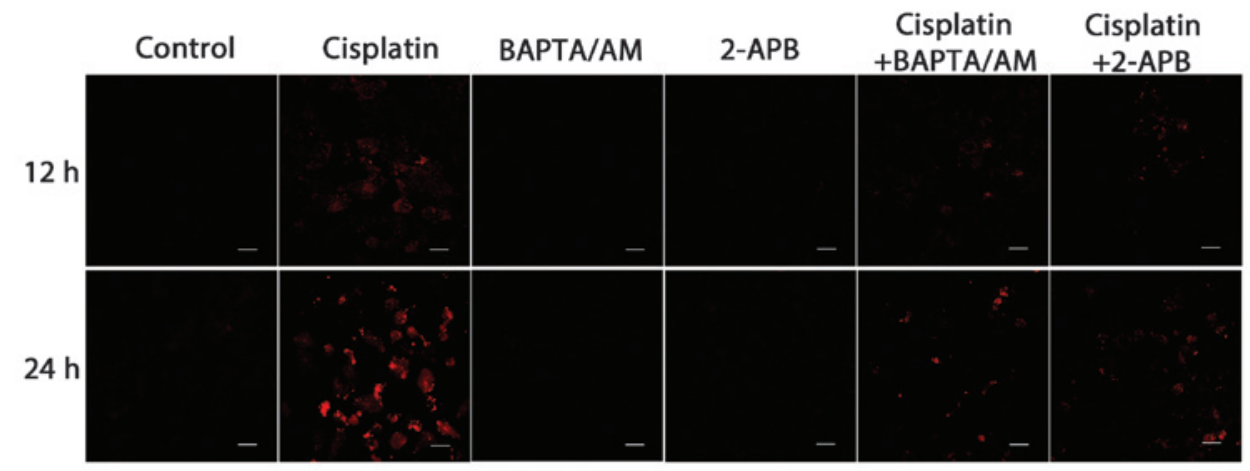

C

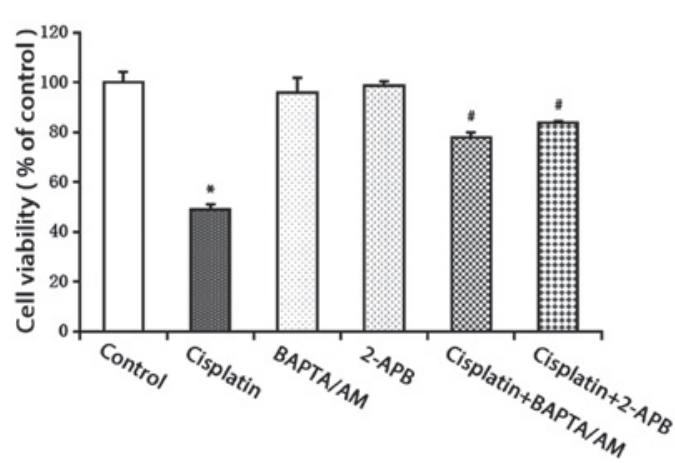

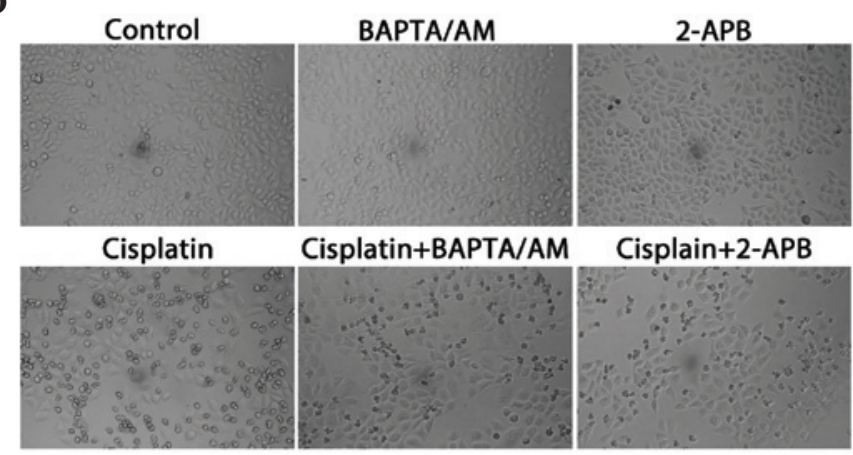

Figure 2. Inhibition of calcium signaling decreases the level of free $\mathrm{Ca}^{2+}$ in the cytosol and mitochondria, and inhibits cell growth. (A) HeLa cells were treated with cisplatin $(5 \mu \mathrm{g} / \mathrm{ml})$ with or without BAPTA/AM $(2.5 \mu \mathrm{M})$ and 2 -APB $(100 \mu \mathrm{M})$ for 12 and $24 \mathrm{~h}$. The cells were incubated with the fluorescent calcium indicator, Fluo-4/AM. Calcium concentrations in the cytosol were observed by confocal microscopy (scale bar, $40 \mu \mathrm{m}$ ). (B) HeLa cells were treated with cisplatin $(5 \mu \mathrm{g} / \mathrm{ml})$ with or without BAPTA/AM $(2.5 \mu \mathrm{M})$ and 2-APB $(100 \mu \mathrm{M})$ for 12 and $24 \mathrm{~h}$, and incubated with the fluorescent calcium indicator, Rhod-2. Calcium concentrations in the mitochondria were observed by confocal microscopy (scale bar, $30 \mu \mathrm{m})$. (C) HeLa cells were treated with cisplatin (5 $\mu \mathrm{g} / \mathrm{ml})$ with or without BAPTA/AM $(2.5 \mu \mathrm{M})$ and 2-APB $(100 \mu \mathrm{M})$ for $24 \mathrm{~h}$. Cell viability was determined using the 3-(4,5-dimetrylthiazol-2-yl)-2,5-diphenyltetrazolium bromide assay. Data are presented as the mean \pm standard deviation $(\mathrm{n}=3)$. ${ }^{*} \mathrm{P}<0.05$ vs. control; ${ }^{\#} \mathrm{P}<0.05$ vs. cisplatin. (D) HeLa cells were treated with cisplatin $(5 \mu \mathrm{g} / \mathrm{ml})$ with or without BAPTA/AM $(2.5 \mu \mathrm{M})$ and 2-APB $(100 \mu \mathrm{M})$ for $24 \mathrm{~h}$. Cell morphology was observed using an inverted phase contrast microscope at x100 magnification. BAPTA/AM, bis-(o-aminophenoxy)ethane-N,N,N',N'-tetra-acetic acid acetoxymethyl ester; 2-APB, 2-aminoethyl diphenylborinate.

Calcium signaling is involved in cisplatin-induced mitochondria-dependent apoptosis. To evaluate the action of calcium in cisplatin-induced apoptotic cell death, the calcium chelating agent, BAPTA/AM, and the IP3R inhibitor, 2-APB, were used to alter the free $\mathrm{Ca}^{2+}$ levels induced by cisplatin. It was observed that the cisplatin-induced free $\mathrm{Ca}^{2+}$ levels in the cytosol and mitochondria substantially decreased in the groups treated with cisplatin combined with BAPTA/AM or 2-APB (Fig. 2A and B).

The MTT assay was used to detect cell viability. The results demonstrated that cisplatin inhibited cell viability in the HeLa cells. Treatment combined with BAPTA/AM or 2-APB decreased the cytotoxic effects of cisplatin $(\mathrm{P}<0.001)$, while treatment with BAPTA/AM or 2-APB alone had no significant effect on cell viability (Fig. 2C). At the same time, an optical microscope was used to examine cellular morphological changes. Compared with the controls, the cells treated with cisplatin became round and fragmented. The numbers of rounded and fragmented cells in the cultures treated with cisplatin combined with BAPTA/AM or 2-APB were less than that in the group treated with only cisplatin (Fig. 2D).

Based on the MTT results, flow cytometry was used to detect the ratio of cell apoptosis induced by cisplatin in the HeLa cells. Consistent with the MTT results, the rate of apoptosis in the cells treated with cisplatin combined with BAPTA/AM or 2-APB (37.8 and $36.1 \%$, respectively) was lower than that of the cells treated only with cisplatin $(55.3 \%)$ (Fig. 3A). Using confocal microscopy, apoptotic chromatin condensation was examined with Hoechst 33342 staining. Treatment with cisplatin alone induced significant apoptotic 
A
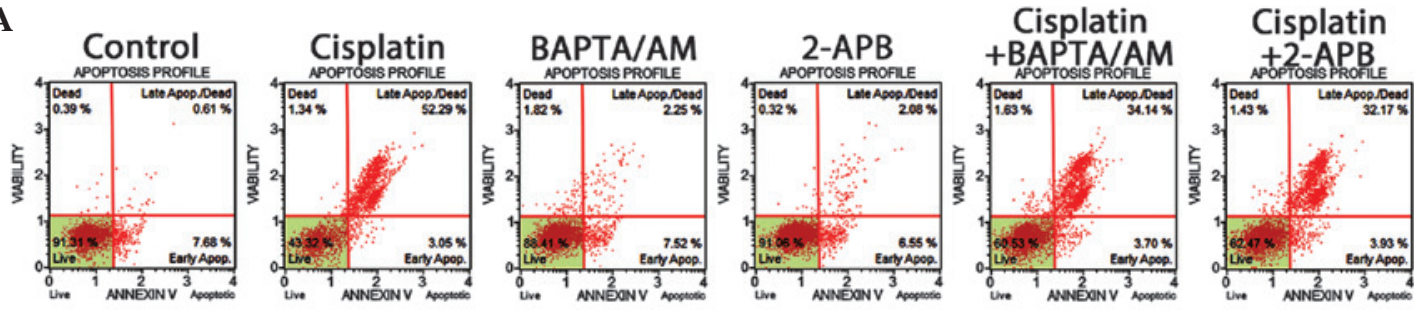

B
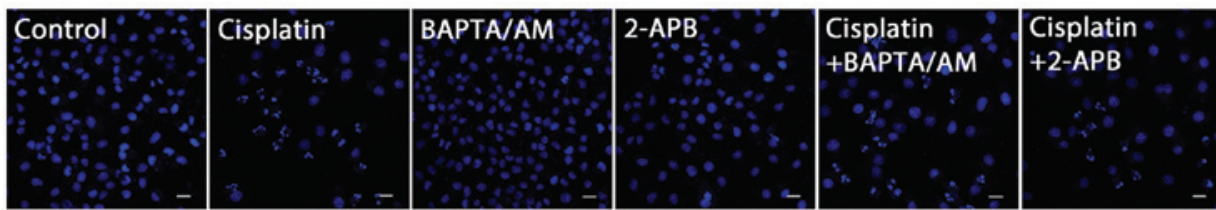

C

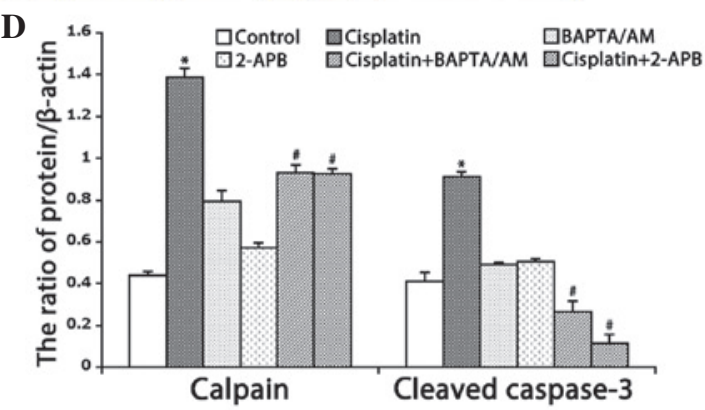

$\mathbf{E}$

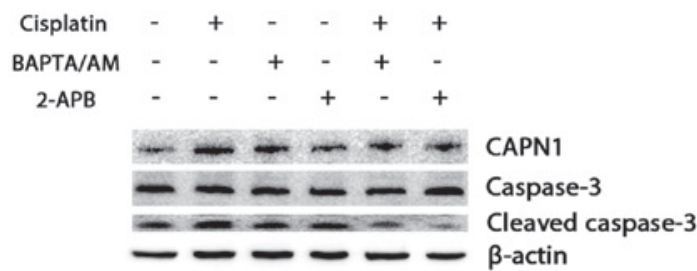

Hoechst-nucleus Active caspase-3 Merge

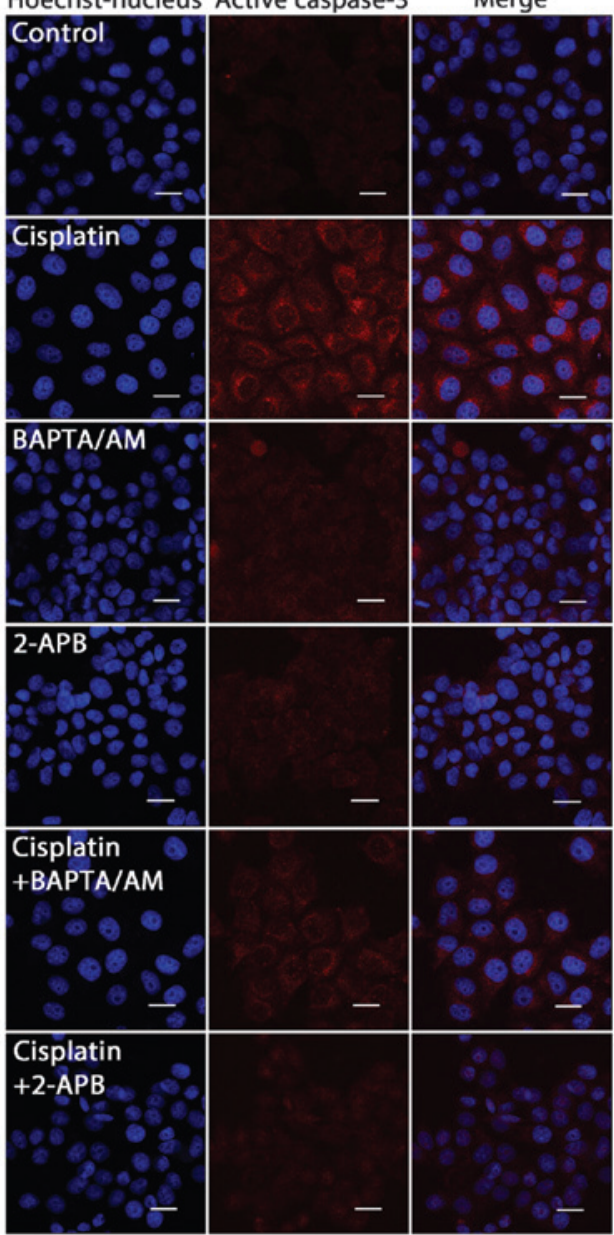

Figure 3. Inhibition of calcium signaling decreases cisplatin-induced mitochondria-mediated apoptosis in HeLa cells. (A) HeLa cells were treated with cisplatin $(5 \mu \mathrm{g} / \mathrm{ml})$ with or without BAPTA/AM $(2.5 \mu \mathrm{M})$ and 2-APB $(100 \mu \mathrm{M})$ for $24 \mathrm{~h}$ and then stained with Annexin-V. Data are presented as the mean $\pm \mathrm{SD}(\mathrm{n}=3)$. (B) HeLa cells were treated with cisplatin $(5 \mu \mathrm{g} / \mathrm{ml})$ with or without BAPTA/AM $(2.5 \mu \mathrm{M})$ and 2-APB $(100 \mu \mathrm{M})$ for $24 \mathrm{~h}$, and stained with Hoechst 33342 . Cell morphology was observed by confocal microscopy (scale bar, $30 \mu \mathrm{m}$ ). (C) The expression of calpain, caspase-3 and cleaved caspase-3 in HeLa cells treated with cisplatin $(5 \mu \mathrm{g} / \mathrm{ml})$ with or without BAPTA/AM $(2.5 \mu \mathrm{M})$ and 2-APB $(100 \mu \mathrm{M})$ for $12 \mathrm{~h}$ by western blotting. (D) Quantitation of calpain and cleaved caspase-3 protein levels. Data are presented as the mean $\pm \mathrm{SD}(\mathrm{n}=3)$. ${ }^{*} \mathrm{P}<0.05$ vs. control; ${ }^{*} \mathrm{P}<0.05$ vs. cisplatin. (E) The expression of cleaved caspase-3 was detected by confocal microscopy following the various treatments for $12 \mathrm{~h}$ (scale bar, $20 \mu \mathrm{m}$ ). BAPTA/AM, bis-(o-aminophenoxy)ethane-N,N,N',N'-tetra-acetic acid acetoxymethyl ester; 2-APB, 2-aminoethyl diphenylborinate; SD, standard deviation. 
A

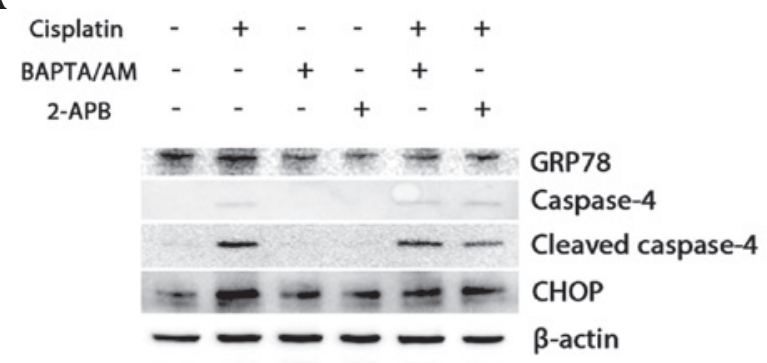

C

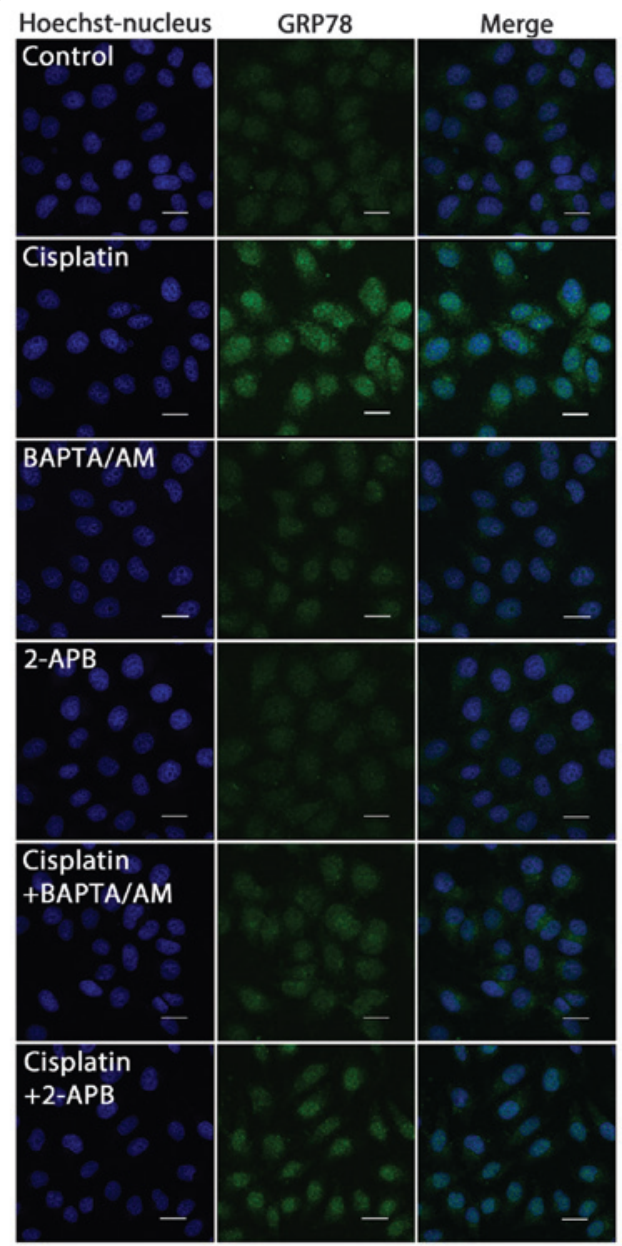

B

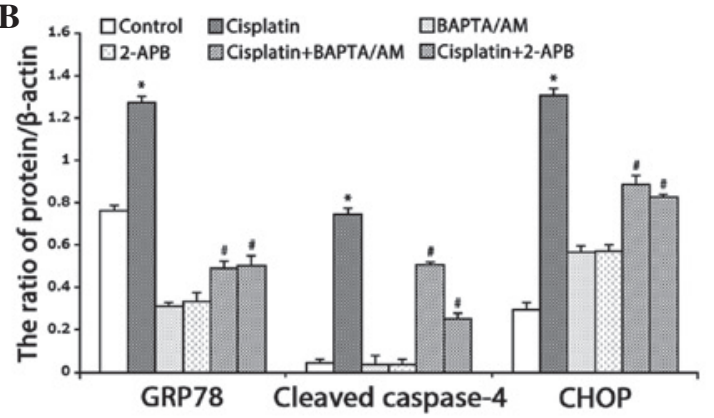

D

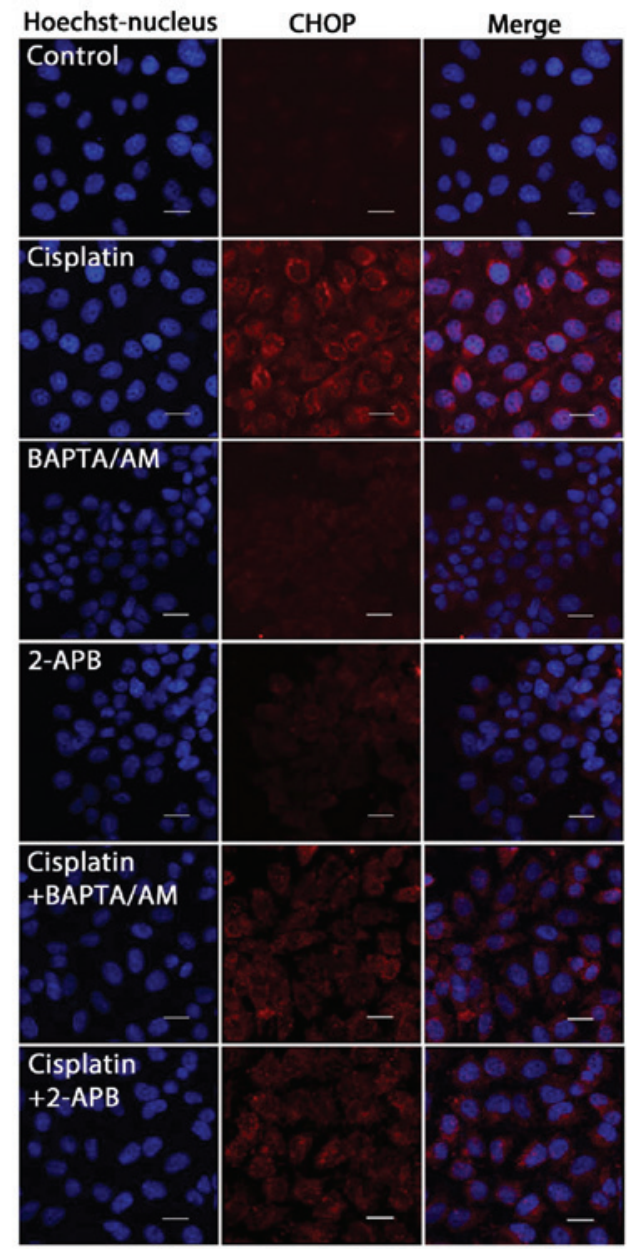

Figure 4. Inhibition of calcium signaling decreases cisplatin-induced ER stress-mediated apoptosis in HeLa cells. (A) Western blotting detection of ER stress proteins in HeLa cells treated with cisplatin $(5 \mu \mathrm{g} / \mathrm{ml})$ with or without BAPTA/AM $(2.5 \mu \mathrm{M})$ and 2-APB (100 $\mu \mathrm{M})$ for $12 \mathrm{~h}$. (B) Quantitation of ER stress protein levels. Data are presented as the mean \pm standard deviation $(n=3)$. ${ }^{*} \mathrm{P}<0.05$ vs. control; ${ }^{\#} \mathrm{P}<0.05$ vs. cisplatin. $(\mathrm{C})$ The expression of GRP78 was detected by confocal microscopy following various treatments for $12 \mathrm{~h}$ (scale bar, $20 \mu \mathrm{m}$ ). (D) The expression of CHOP was detected by confocal microscopy following various treatments for $12 \mathrm{~h}$ (scale bar, $20 \mu \mathrm{m}$ ). BAPTA/AM, bis-(o-aminophenoxy)ethane-N,N,N',N'-tetra-acetic acid acetoxymethyl ester; 2-APB, 2-aminoethyl diphenylborinate; CHOP, C/EBP homologous protein; GRP78, glucose-regulated protein, 78 kDa; ER, endoplasmic reticulum.

chromatin condensation in the HeLa cells, while the cells treated with cisplatin combined with BAPTA/AM or 2-APB showed a lower ratio of apoptotic chromatin condensation (Fig. 3B).

CAPN1 (also known as $\mu$-calpain) is a $\mathrm{Ca}^{2+}$-dependent cysteine protease, and its activation requires high levels of free calcium in the cytosol $(20,21)$. Thus, the present study assessed calcium levels and apoptosis in the HeLa cells by monitoring the expression of CAPN1 and cleaved caspase- 3 by western blotting. As shown in Fig. 3C and D, cisplatin enhanced the expression of CAPN1 and cleaved caspase-3
$(\mathrm{P}<0.001)$. Meanwhile, treatment with cisplatin combined with BAPTA/AM or 2-APB decreased the level of CAPN1 and cleaved caspase- 3 induced by cisplatin. Consistent with the western blotting results, immunofluorescence against caspase-3 revealed a similar effect (Fig. 3E). These results indicated that cisplatin upregulates intracellular calcium mobilization and initiates apoptotic events.

Cisplatin-induced pro-apoptosis calcium signaling is derived from ER stress. Cisplatin has been reported to induce ER stress and downstream processes associated with apoptosis $(7,10,22)$. 
To further verify if the resultant apoptosis was partially induced by ER stress, the present study assessed the occurrence of ER stress and ER-associated apoptosis by examining the expression levels of ER stress-associated proteins.

The expression of GRP78, an ER molecular chaperone that induces ER stress upon accumulation, was detected. Western blotting revealed that cisplatin upregulated the expression of GRP78, and that combined treatment with BAPTA/AM or 2-APB downregulated the expression of GRP78 (Fig. 4A and B; $\mathrm{P}<0.001)$. Using confocal microscopy, it was observed that the accumulation of GRP78 increased in cells treated with cisplatin only, while combined treatment with BAPTA/AM or 2-APB markedly decreased the cisplatin-induced GRP78 accumulation (Fig. 4C).

Next, the ER stress-associated apoptotic proteins, caspase-4 and CHOP, were monitored. Western blotting revealed that cisplatin enhanced the expression of CHOP and cleaved caspase-4, while combined treatment with BAPTA/AM or 2-APB inhibited these effects (Fig. 4A and B; P<0.001). Similarly, confocal microscopy revealed marked accumulation of CHOP in cells treated with cisplatin alone, and low-level CHOP accumulation in groups treated with cisplatin combined with BAPTA/AM or 2-APB (Fig. 4D).

These results demonstrated that cisplatin induced pro-apoptotic calcium signaling, which resulted in ER stress-mediated apoptotic events in the HeLa cells.

\section{Discussion}

Cisplatin is one of several effective antitumor drugs widely used against multiple solid tumors in the clinic; it acts by damaging DNA, inhibiting DNA synthesis and inducing apoptosis in cancer cells $(5,23)$. Previous studies have reported that chemotherapy drugs, including cisplatin, cause mitochondrial dysfunction, the release of cytochrome $c$, the activation of the caspase-mediated cascade and apoptotic cell death (24-27). In the present study, it was found that cisplatin inhibited cell growth, upregulated active caspase- 3 and induced the mitochondria-mediated apoptosis pathway in human HeLa cells (Figs. 2 and 3). An increasing amount of evidence has indicated that cisplatin induces cell apoptosis through ER stress $(9,10,22)$. The ER is an important organelle in eukaryotic cells, and is involved in several critical processes. Under stressful conditions, numerous unfolded and incompletely folded proteins accumulate in the ER lumen, which stimulates the UPR, leading to ER stress $(11,12)$. It is generally considered that the accumulation of the calcium-dependent molecular chaperone, GRP78, initiates ER stress $(10,28)$. The results of the present study indicated that treatment with cisplatin induces the accumulation of GRP78 in HeLa cells and results in ER stress (Fig. 4A and C). The transcription factor CHOP (also known as DNA damage-inducible transcript 3) is an ER stress-associated apoptosis protein that integrates the apoptotic endoplasmic reticulum to nucleus signaling 1, eukaryotic translation initiation factor $2 \alpha$ kinase 3 and activating transcription factor 6 pathways in the UPR (29-32). Caspase- 4 is a calcium-dependent caspase; its overexpression stimulates the downstream cascade reaction and leads to cell apoptosis, which is caused by destruction of the ER and calcium pool emptying (33-35). Thus, the present study examined the expression of these two ER stress-associated apoptotic proteins. The results demonstrated that cisplatin markedly increased the expression levels of these two proteins (Fig. 4A and B). These data suggested that in addition to the mitochondria-mediated apoptotic pathway, cisplatin could also induce cell apoptosis via ER stress. Notably, concurrently with the occurrence of apoptosis, the cytosolic and mitochondrial free $\mathrm{Ca}^{2+}$ levels increased, and calpain expression was upregulated. The modulation of calcium concentration in the cytoplasm affected the activation of the calpains, which are evolutionarily conserved $\mathrm{Ca}^{2+}$-dependent cysteine proteases $(36,37)$. It is reported that the overexpression of calpains promotes the cleavage of Bid, which in turn modulates Bax and activates caspase- 3 . There is also evidence to suggest that activation of caspase- 4 requires calpains (38-40). The aforementioned results suggest that calcium signaling involves two apoptotic pathways: i) Calcium ion entry into the mitochondria, which induces calcium disorder in he mitochondria and leads to mitochondria-mediated apoptosis; and ii) calcium signaling in the cytosol activates calcium-dependent proteases and results in the expression of ER stress-associated apoptotic proteins.

To investigate this hypothesis, the calcium chelating agent, BAPTA/AM, was used to block free $\mathrm{Ca}^{2+}$ in the cytoplasm. Won et al reported that apoptosis induced by $3 \alpha, 23$-isopropylidenedioxyolean-12-en-27-oic acid was substantially reduced following combined treatment with BAPTA/AM in HeLa cells (40). In the present study, HeLa cells were treated with cisplatin combined with BAPTA/AM. The data demonstrated that calcium concentrations in the cytoplasm and expression levels of calpain were reduced in the presence of BAPTA/AM. Moreover, in the group treated with cisplatin combined with BAPTA/AM, the ER-stress proteins CHOP and active caspase-4 were downregulated (Fig. 4A and B). These results indicated that endogenous calcium efflux participates in ER stress-mediated apoptosis induced by cisplatin in HeLa cells. Intercellular calcium homeostasis is controlled by calcium ion channels on the ER membrane, which include IP3R, ryanodine receptors and sarcoendoplasmic reticular $\mathrm{Ca}^{2+}$-ATPase pumps $(41,42)$. To further confirm the source of calcium signaling, the IP3R inhibitor, 2-APB, was used to inhibit calcium release from the ER. Yoon et al reported that 2-APB potently inhibited calcium release from the ER and suppressed celastrol-induced apoptosis in breast cancer cell lines (MDA-MB 435S and MCF-7) (43). In the present study, it was found that combined treatment with 2-APB decreased calcium release from the ER and inhibited cisplatin-induced apoptosis in the HeLa cells. The data revealed that these inhibitory effects mainly manifested via two mechanisms: i) 2-APB inhibits calcium flux from the ER to the mitochondria and decreases the expression of cleaved caspase- 3 , which attenuates mitochondria-mediated apoptosis; and ii) 2-APB blocks calcium flux from the ER to the cytosol, inhibits the activation of calpains, and downregulates the expression of the ER stress proteins, $\mathrm{CHOP}$ and caspase- 4 . These results suggested that blocking calcium release from the ER effectively mitigates the ER stress-associated apoptotic pathway. Previous studies have revealed that cisplatin initiates ER stress and interferes with calcium homeostasis $(44,45)$. The present study found that blocking calcium efflux decreased the level of ER stress 
correspondingly. This phenomenon suggests that calcium signaling may act as a positive feedback mechanism by modulating ER stress. However, further investigations are required.

In summary, the present study demonstrated that cisplatin induced ER stress and led to apoptosis in human HeLa cells. Notably, treatment with cisplatin combined with BAPTA/AM or 2-APB markedly decreased the inhibition of cell growth and the rate of apoptosis. The results demonstrate that calcium efflux from the ER regulates cell apoptosis triggered by cisplatin in HeLa cells. In addition, the study provided further mechanistic insights into the tumor cell-killing effect of cisplatin and potential therapeutic strategies to improve cisplatin chemotherapy.

\section{Acknowledgements}

This research was supported by the National Natural Science Foundation of China (grant nos. 81272876 and 81372793) and the Department of Education of Jilin Province Project (grant no. 2013361).

\section{References}

1. Reedijk J: New clues for platinum antitumor chemistry: Kinetically controlled metal binding to DNA. Proc Natl Acad Sci USA 100: 3611-3616, 2003.

2. Wozniak K and Blasiak J: Recognition and repair of DNA-cisplatin adducts. Acta Biochim Pol 49: 583-596, 2002.

3. Koberle B, Tomicic MT, Usanova S and Kaina B: Cisplatin resistance: Preclinical findings and clinical implications. Biochim Biophys Acta 1806: 172-182, 2010.

4. Basu A and Krishnamurthy S: Cellular responses to Cisplatin-induced DNA damage. J Nucleic Acids 2010: pii; 201367, 2010.

5. Terada $Y$, Inoue $\mathrm{K}$, Matsumoto $\mathrm{T}$, Ishihara M, Hamada $\mathrm{K}$, Shimamura Y, Ogata K, Inoue K, Taniguchi Y, Horino T, et al 5-Aminolevulinic acid protects against cisplatin-induced nephrotoxicity without compromising the anticancer efficiency of cisplatin in rats in vitro and in vivo. PloS One 8: e80850, 2013.

6. Maimaitiyiming H, Li Y, Cui W, Tong X, Norman H, Qi X and Wang S: Increasing cGMP-dependent protein kinase I activity attenuates cisplatin-induced kidney injury through protection of mitochondria function. Am J Physiol Renal Physiol 305: F881-F890, 2013.

7. Jiang $M$, Wang CY, Huang $S$, Yang $T$ and Dong $Z$ : Cisplatin-induced apoptosis in p53-deficient renal cells via the intrinsic mitochondrial pathway. Am J Physiol Renal Physiol 296: F983-F993, 2009.

8. Xu Y, Wang C and Li Z: A new strategy of promoting cisplatin chemotherapeutic efficiency by targeting endoplasmic reticulum stress. Mol Clin Oncol 2: 3-7, 2014.

9. Xu Y, Yu H, Qin H, et al: Inhibition of autophagy enhances cisplatin cytotoxicity through endoplasmic reticulum stress in human cervical cancer cells. Cancer Lett 314: 232-243, 2012.

10. Kim I, Xu W and Reed JC: Cell death and endoplasmic reticulum stress: Disease relevance and therapeutic opportunities. Nature reviews. Nat Rev Drug Discov 7: 1013-1030, 2008.

11. Schröder M and Kaufman RJ: ER stress and the unfolded protein response. Mutat Res 569: 29-63, 2005.

12. Smedler E and Uhlén P: Frequency decoding of calcium oscillations. Biochim Biophys Acta 1840: 964-969, 2014.

13. Torres M, Encina G, Soto C and Hetz C: Abnormal calcium homeostasis and protein folding stress at the ER: A common factor in familial and infectious prion disorders. Commun Integr Biol. 4: 258-261, 2011.

14. Mekahli D, Bultynck G, Parys JB, De Smedt H and Missiaen L: Endoplasmic-reticulum calcium depletion and disease. Cold Spring Harb Perspect Biol 3: pii, a004317, 2011.

15. Rharass T, Lemcke H, Lantow M, Kuznetsov SA, Weiss DG and Panáková $\mathrm{D}: \mathrm{Ca}^{2+}$-mediated mitochondrial reactive oxygen species metabolism augments $\mathrm{Wnt} / \beta$-catenin pathway activation to facilitate cell differentiation. J Biol Chem 289: 27937-27951, 2014.
16. Tusskorn O, Senggunprai L, Prawan A, Kukongviriyapan U and Kukongviriyapan V: Phenethyl isothiocyanate induces calcium mobilization and mitochondrial cell death pathway in cholangiocarcinoma KKU-M214 cells. BMC Cancer 13: 571, 2013.

17. Roy SS and Hajnóczky G: Calcium, mitochondria and apoptosis studied by fluorescence measurements. Methods 46: 213-223, 2008.

18. Burkeen JF, Womac AD, Earnest DJ and Zoran MJ: Mitochondrial calcium signaling mediates rhythmic extracellular ATP accumulation in suprachiasmatic nucleus astrocytes. J Neurosci 31: 8432-8440, 2011

19. Goll DE, Thompson VF, Li H, Wei W and Cong J: The calpain system. Physiol Rev 83: 731-801, 2003.

20. Neuhof $\mathrm{C}$ and Neuhof $\mathrm{H}$ : Calpain system and its involvement in myocardial ischemia and reperfusion injury. World J Cardiol 6: 638-652, 2014.

21. Suzuki M, Endo M, Shinohara F, Echigo S and Rikiishi H: Enhancement of cisplatin cytotoxicity by SAHA involves endoplasmic reticulum stress-mediated apoptosis in oral squamous cell carcinoma cells. Cancer Chemother Pharmacol 64: 1115-1122, 2009.

22. Gonzalez VM, Fuertes MA, Alonso C and Perez JM: Is cisplatin-induced cell death always produced by apoptosis? Mol Pharmacol 59: 657-663, 2001

23. Strasser A, O'Connor L and Dixit VM: Apoptosis signaling. Annu Rev Biochem 69: 217-245, 2000.

24. Zhang J, Kamdar O, Le W, Rosen GD and Upadhyay D: Nicotine induces resistance to chemotherapy by modulating mitochondrial signaling in lung cancer. Am J Respir Cell Mol Biol 40: 135-146, 2009.

25. Jin L, Tabe Y, Kojima K, Zhou Y, Pittaluga S, Konopleva M, Miida T and Raffeld M: MDM2 antagonist Nutlin-3 enhances bortezomib-mediated mitochondrial apoptosis in TP53-mutated mantle cell lymphoma. Cancer Lett 299: 161-170, 2010.

26. Cullen KJ, Yang Z, Schumaker L and Guo Z: Mitochondria as a critical target of the chemotherapeutic agent cisplatin in head and neck cancer. J Bioenerg Biomembr 39: 43-50, 2007.

27. Zhong JT, Xu Y, Yi HW, Su J, Yu HM, Xiang XY, Li XN, Zhang ZC and Sun LK: The BH3 mimetic S1 induces autophagy through ER stress and disruption of Bcl-2/Beclin 1 interaction in human glioma U251 cells. Cancer Lett 323: 180-187, 2012.

28. Scheuner D, Song B, McEwen E, Liu C, Laybutt R, Gillespie P, Saunders T, Bonner-Weir S and Kaufman RJ: Translational control is required for the unfolded protein response and in vivo glucose homeostasis. Mol Cell 7: 1165-1176, 2001.

29. Oyadomari S and Mori M: Roles of CHOP/GADD153 in endoplasmic reticulum stress. Cell Death Differ 11: 381-389, 2004.

30. Harding HP, Novoa I, Zhang Y, Zeng H, Wek R, Schapira M and Ron D: Regulated translation initiation controls stress-induced gene expression in mammalian cells. Mol Cell 6: 1099-1108, 2000.

31. Tabas I and Ron D: Integrating the mechanisms of apoptosis induced by endoplasmic reticulum stress. Nat Cell Biol 13: 184-190, 2011.

32. Tatsuta T, Hosono M, Miura Y, Sugawara S, Kariya Y, Hakomori S and Nitta K: Involvement of ER stress in apoptosis induced by sialic acid-binding lectin (leczyme) from bullfrog eggs. Int J Oncol 43: 1799-1808, 2013.

33. Li C, Wei J, Li Y, He X, Zhou Q, Yan J, Zhang J, Liu Y, Liu Y and Shu HB: Transmembrane Protein 214 (TMEM214) mediates endoplasmic reticulum stress-induced caspase 4 enzyme activation and apoptosis. J Biol Chem 288: 17908-17917, 2013.

34. Hitomi J, Katayama T, Eguchi Y, Kudo T, Taniguchi M, Koyama Y, Manabe T, Yamagishi S, Bando Y, Imaizumi K, et al: Involvement of caspase-4 in endoplasmic reticulum stress-induced apoptosis and Abeta-induced cell death. J Cell Biol 165: 347-356, 2004.

35. Tompa P, Töth-Boconádi R and Friedrich P: Frequency decoding of fast calcium oscillations by calpain. Cell Calcium 29: 161-170, 2001.

36. Angka L, Lee EA, Rota SG, Hanlon T, Sukhai M, Minden M, McMillan EM, Quadrilatero J and Spagnuolo PA: Glucopsychosine increases cytosolic calcium to induce calpain-mediated apoptosis of acute myeloid leukemia cells. Cancer Lett 348: 29-37, 2014.

37. Sharma AK and Rohrer B: Calcium-induced calpain mediates apoptosis via caspase-3 in a mouse photoreceptor cell line. J Biol Chem 279: 35564-35572, 2004.

38. Mandic A, Viktorsson K, Strandberg L, Heiden T, Hansson J, Linder S and Shoshan MC: Calpain-mediated Bid cleavage and calpain-independent Bak modulation: Two separate pathways in cisplatin-induced apoptosis. Mol Cell Biol 22: 3003-3013, 2002. 
39. Matsuzaki S, Hiratsuka T, Kuwahara R, Katayama $T$ and Tohyama M: Caspase- 4 is partially cleaved by calpain via the impairment of $\mathrm{Ca}^{2+}$ homeostasis under the ER stress. Neurochem Int 56: 352-356, 2010.

40. Won SJ, Ki YS, Chung KS, Choi JH, Bae KH and Lee KT: $3 \alpha, 23$-isopropylidenedioxyolean-12-en-27-oic acid, a triterpene isolated from Aceriphyllum rossii, induces apoptosis in human cervical cancer HeLa cells through mitochondrial dysfunction and endoplasmic reticulum stress. Biol Pharm Bull 33: 1620-1626, 2010.

41. Clapham DE: Calcium signaling. Cell 131: 1047-1058, 2007.

42. Gerasimenko JV, Gerasimenko OV and Petersen OH: The role of $\mathrm{Ca}^{2+}$ in the pathophysiology of pancreatitis. J Physiol 592: 269-280, 2014
43. Yoon MJ, Lee AR, Jeong SA, Kim YS, Kim JY, Kwon YJ and Choi KS: Release of $\mathrm{Ca}^{2+}$ from the endoplasmic reticulum and its subsequent influx into mitochondria trigger celastrol-induced paraptosis in cancer cells. Oncotarget 5: 6816-6831, 2014.

44. Tian F, Schrödl K, Kiefl R, Huber RM and Bergner A: The hedgehog pathway inhibitor GDC-0449 alters intracellular $\mathrm{Ca}^{2+}$ homeostasis and inhibits cell growth in cisplatin-resistant lung cancer cells. Anticancer Res 32: 89-94, 2012.

45. Chen Y, Tsai YH and Tseng SH: RECK regulated endoplasmic reticulum stress response and enhanced cisplatin-induced cell death in neuroblastoma cells. Surgery 154: 968-979, 2013. 